\title{
Ultrafast cone-beam CT scatter correction with GPU-based Monte Carlo simulation
}

\author{
Yuan Xu, Ti Bai, Hao Yan, Luo Ouyang, Jing Wang, Arnold Pompos, Linghong Zhou, \\ Steve B Jiang, Xun Jia
}

Department of Radiation Oncology, UT Southwestern Medical Center, Dallas, TX, USA.

Received March 19, 2014; Published Online April 08, 2014

[Presented at the Young Investigator's Symposium at the 2014 Annual Meeting of

Southwest Chapter of American Association of Physicists in Medicine (AAPM) in San Antonio, Texas, USA]

\section{Conference Proceeding}

\begin{abstract}
Purpose: Scatter artifacts severely degrade image quality of cone-beam CT (CBCT). We present an ultrafast scatter correction framework by using GPU-based Monte Carlo (MC) simulation and prior patient CT image, aiming at automatically finish the whole process including both scatter correction and reconstruction within 30 seconds.
\end{abstract}

Methods: The method consists of six steps: 1) FDK reconstruction using raw projection data; 2) Rigid Registration of planning CT to the FDK results; 3) MC scatter calculation at sparse view angles using the planning CT; 4) Interpolation of the calculated scatter signals to other angles; 5) Removal of scatter from the raw projections; 6) FDK reconstruction using the scatter-corrected projections. In addition to using GPU to accelerate MC photon simulations, we also use a small number of photons and a down-sampled CT image in simulation to further reduce computation time. A novel denoising algorithm is used to eliminate $\mathrm{MC}$ noise from the simulated scatter images caused by low photon numbers. The method is validated on one simulated head-and-neck case with 364 projection angles.

Results: We have examined variation of the scatter signal among projection angles using Fourier analysis. It is found that scatter images at 31 angles are sufficient to restore those at all angles with $<0.1 \%$ error. For the simulated patient case with a resolution of $512 \times 512 \times 100$, we simulated $5 \times 10^{6}$

Presenting author: Yuan Xu; Department of Radiation Oncology, UT Southwestern Medical Center, Dallas, TX, USA.

Cite this article as:

Xu Y, Bai T, Yan H, Ouyang L, Wang J, Pompos A, Zhou L, Jiang

$\mathrm{SB}$, Jia X. Ultrafast cone-beam CT scatter correction with GPU-based Monte Carlo simulation. Int J Cancer Ther Oncol 2014; 2(2):020245. DOI: 10.14319/ijcto.0202.45 photons per angle. The total computation time is 20.52 seconds on a Nvidia GTX Titan GPU, and the time at each step is $2.53,0.64,14.78,0.13,0.19$, and 2.25 seconds, respectively. The scatter-induced shading/cupping artifacts are substantially reduced, and the average HU error of a region-of-interest is reduced from 75.9 to $19.0 \mathrm{HU}$.

Conclusion: A practical ultrafast MC-based CBCT scatter correction scheme is developed. It accomplished the whole procedure of scatter correction and reconstruction within 30 seconds.

Innovation/Impact: While Monte Carlo-based CBCT scatter estimation and removal has been proposed for a long time, hardly can we realize this in real clinical workflow due to the long computation time. Our work, for the first time, achieves a high computation efficiency (30 sec) using novel GPU-based MC techniques and numerical methods, making MC-based scatter removal possible in clinic. 


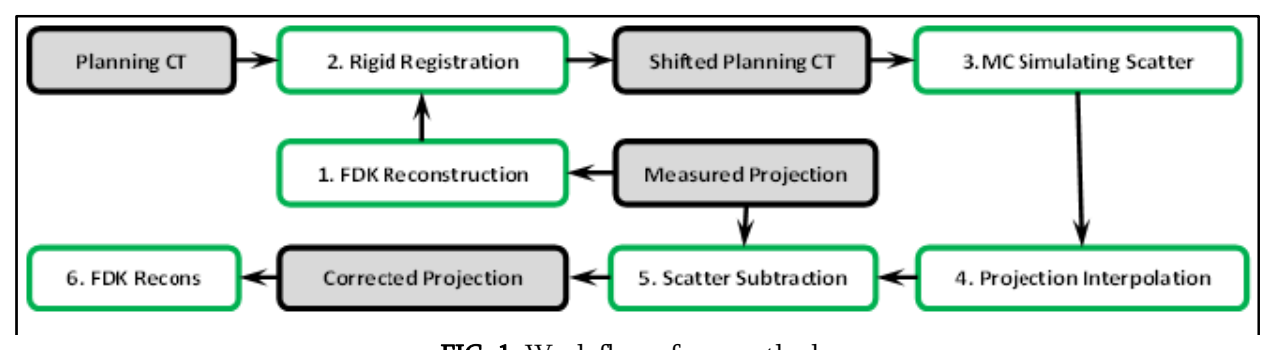

FIG. 1: Work flow of our method.

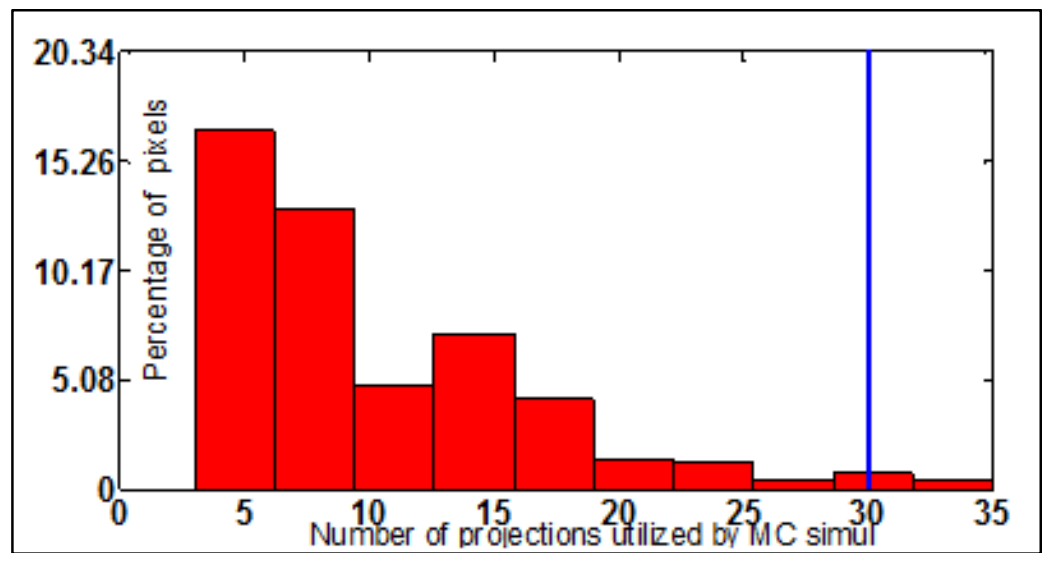

FIG.2: The histogram of minimum frequency needed to restore scatter pixel values at all angles.
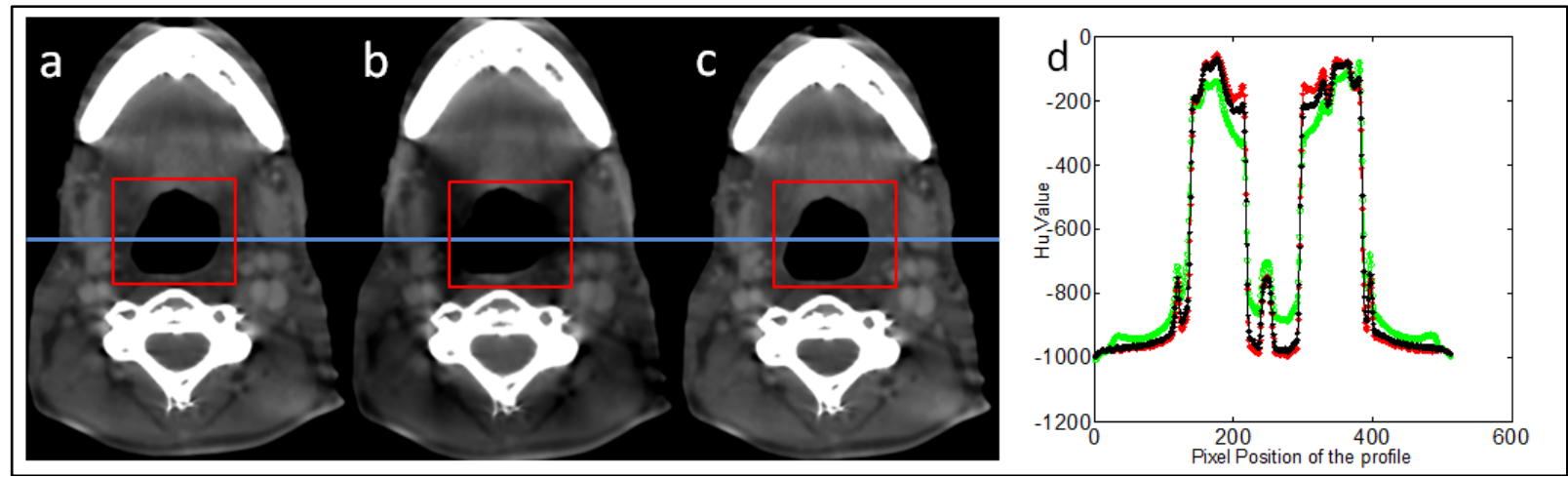

FIG. 3: a)-c) FDK results with primal projection only (ground truth), with scatter-contaminated projections, and scatter corrected projections. c) Black, green, red lines are profiles of the horizontal blue line in a), b), c) ,respectively. 